\title{
La macroglossia: un'altra complicanza dei pazienti con oltre 20 anni di dialisi
}

\author{
O. Marzolla ${ }^{1}$, V. Candela ${ }^{1}$, M.L. Mannino ${ }^{1}$, S.A. Malara ${ }^{1}$, C. Labate ${ }^{1}$, \\ M. Garozzo ${ }^{2}$, G. Enia ${ }^{2}$
}

\section{${ }^{1}$ Unità Operativa di Nefrologia e Dialisi ASL n. 11 - Melito Porto Salvo e Scilla ${ }^{2}$ Divisione Nefrologica \& Centro di Fisiologia Clinica del CNR - Reggio Calabria}

noto che l'amiloidosi primitiva può: interessare la lingua determianndo macroglossia (1). Questa complicanza è invece molto rara nelle amiloidosi secondarie ed è considerata evenienza eccezionale nell'amiloidosi dialitica (2). Nella banca dati Medline, è registrato un unico paziente affetto da amiloidosi dialitica che aveva sviluppato macroglossia, descritto da Sethi nel 1990 (3). La nostra recente esperienza, che qui riportiamo, sembra indicare che l'amiloidosi dialitica di lunga durata determini macroglossia più frequentemente di quanto si creda.

Nell'ultimo anno abbiamo osservato, su una popolazione di 115 pazienti in emodialisi, 2 casi di grave macroglossia (Figg. 1 e 2). La prevalenza di questa complicanza era del 18\% nel sottogruppo dei pazienti che avevano superato $\mathrm{i}$ 20 anni di trattamento dialitico $(n=11)$, e raggiungeva il $40 \%$ nei pazienti in trattamento da 25 o più anni $(n=5)$.

I due pazienti che avevano sviluppato macroglossia erano stati in emodialisi rispettivamente per 26 e 25 anni ed avevano effettuato il trattamento, quasi esclusivamente, con filtri di Cuprophan. Uno di essi era stato trattato per 66 mesi mediante la CAPD per poi tornare in emodialisi. Entrambi i pazienti avevano una gravissima forma di amiloidosi osteoarticolare ed erano stati operati per sindrome del tunnel carpale bilaterale. La macroglossia è stata osservata dopo

circa 8 anni dal primo intervento, ed è stata diagnosticata per la comparsa di una discreta difficoltà nella deglutizio-

Fig. 1 - Paziente di 56 anni in emodialisi dal 1971.

Fig. 2 - Paziente di 57 anni in emodialisi dal 1972.
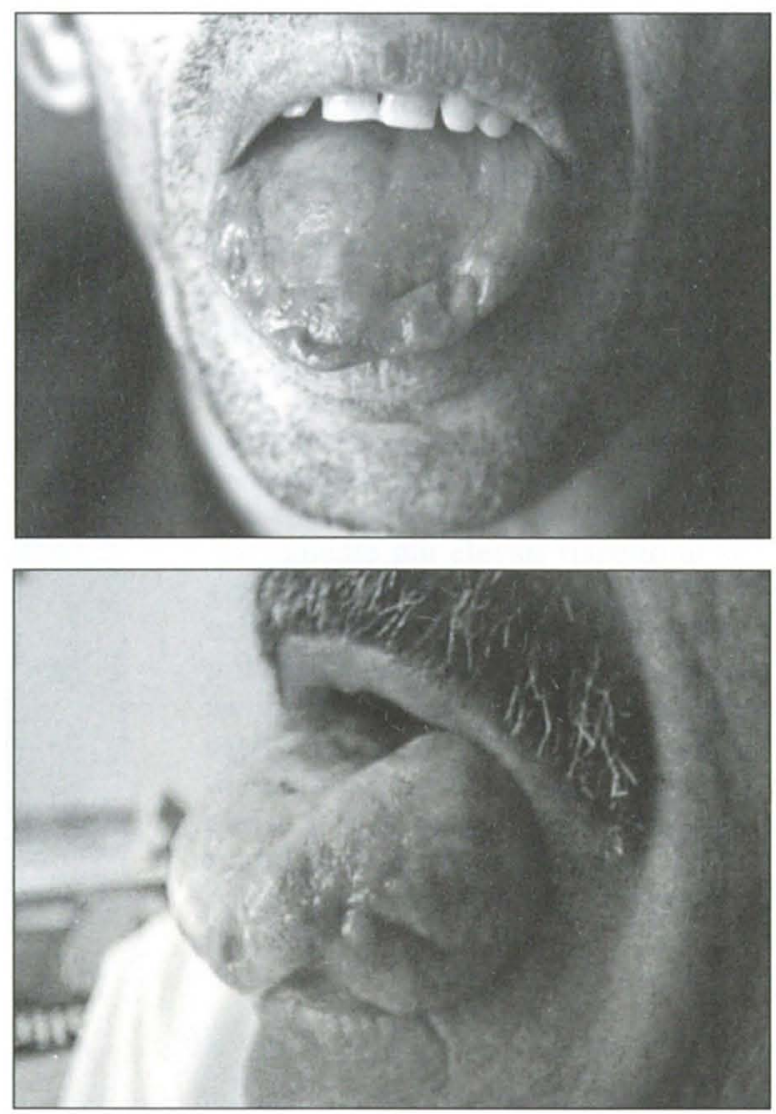
ne, che, in un paziente, contribuiva ad aggravare un importante stato di malnutrizione. L'esame istologico mostrava in entrambi i casi la presenza di depositi di amiloide.

La nostra esperienza dimostra che la macroglossia è una complicanza tardiva nella storia naturale dell'amiloidosi dialitica che può interessare oltre $1 / 3$ dei pazienti che hanno superato i 25 anni di dialisi.

È infine da rilevare che un recente studio giapponese ha evidenziato nell' $8 \%$ dei pazienti con 20 o più anni di trattamento dialitico, la presenza sulla lingua di piccoli noduli bianco-giallastri di amiloide senza segni di macroglossia (2). I nostri 2 casi, insieme a quello descritto da Sethi nel Regno Unito (3), sembrano indicare che alcune manifestazioni cliniche dell'amiloidosi dialitica nei pazienti caucasici possono differire da quelle degli asiatici.

\section{BIBLIOGRAFIA}

1. Cohen AS. Amyloidosis. In: Isselbacher KJ, Braunwald E, Wilson JD, et al. Harrison's Principles of Internal Medicine (Eds). Mc Graw-Hill, New York, 1994; 162530.

2. Matsuo K, Nakamoto M, Yasunaga $\mathrm{C}$, et al. Dialysis-related amyloidosis of the tongue in longterm hemodialysis patients. Kidney Int 1997; 52: 832-8.

3. Sethi D, Hutchison AJ, Cary NRB, et al. Macroglossia and amyloidoma of the buttock: evidence of systemic involvement in dialysis amyloid. Nephron 1990; 55: 312-5. 